\title{
Alterner les langues dans les MOOC Enjeux pour la transmission et la transformation des savoirs
}

\section{Alternating languages in MOOC: issues for the transmission and transformation of knowledge}

\section{Alternancia de lenguas en los MOOC: retos para la transmisión y transformación del conocimiento}

https://doi.org/10.52358/mm.vi7.223

Laurent Gajo, professeur ordinaire

Université de Genève, Suisse

laurent.gajo@unige.ch

Mariana Fonseca Favre, maitre-assistante

Université de Genève, Suisse

mariana.fonseca@unige.ch

Gabriela Steffen, collaboratrice scientifique

Université de Genève, Suisse

gabriela.steffen@unige.ch

\section{RÉSUMÉ}

Les $M O O C$, dans leur ambition de transmission des savoirs à large échelle, privilégient les langues à grande diffusion, souvent l'anglais, et fonctionnent souvent dans une seule langue. Dans cet article, nous remettons en question le rôle du plurilinguisme dans les MOOC et, en particulier, le mécanisme de l'alternance codique, qui peut intervenir à divers niveaux et concerne notamment la place et l'usage du sous-titrage. En croisant les regards des concepteurs, des conceptrices, des usagers, des usagères et des linguistes, nous décrivons le potentiel du plurilinguisme non seulement pour la communication des savoirs, mais pour leur problématisation. Notre analyse permet de revenir sur la tension entre les processus d'universalisation et de contextualisation. 
Mots-clés : transmission et construction des connaissances, alternance codique, MOOC, plurilinguisme, sous-titrage

\section{ABSTRACT}

As MOOCs aim to transmit knowledge on a large scale, they tend to prefer widely used languages such as English and operate in a single language. This article discusses the role of plurilingualism in MOOCs and the mechanism of code-switching in particular, which can occur at various levels and involve, for instance, the place and use of subtitling. It describes the potential of plurilingualism not only for communicating knowledge but also for problematizing it, crossing the views of designers, users and linguists. This analysis offers scope for revisiting the tension between the processes of globalization and contextualization.

Keywords: knowledge transmission and construction, language alternation, MOOCs, plurilingualism, subtitling

\section{RESUMEN}

Los MOOC, en su ambición de transmitir conocimientos a gran escala, favorecen a las lenguas con mayor difusión, a menudo el inglés, y suelen operar en un solo idioma. En este artículo, cuestionamos el papel del plurilingüismo en los MOOC y, en particular, el mecanismo de alternancia de códigos, que puede darse en varios niveles y que concierne, particularmente, la presencia y el uso de subtítulos. Cruzando los puntos de vista de diseñadores/as, usuarios/as y lingüistas, describimos el potencial del plurilingüismo no solamente para la transmisión del conocimiento, sino también para su problematización. Nuestro análisis nos permite tratar de nuevo la tensión existente entre los procesos de universalización y contextualización.

Palabras clave: transmisión y construcción del conocimiento, alternancia de códigos, MOOC, plurilingüismo, subtitulado

\section{Introduction}

Les MOOC présentent un potentiel énorme de diffusion des savoirs. L'accessibilité de ces derniers demande toutefois d'interroger les compétences langagières des usagers et usagères, et leur ancrage culturel. Dans ce cadre, la question du plurilinguisme et, en particulier, du sous-titrage dans les MOOC prend une pertinence croissante (Fonseca et Gajo, 2020).

Le processus de sous-titrage interpelle plus largement le processus de médiation (Cavalli et Coste, 2019) dans au moins deux directions : le passage des savoirs d'une figure experte à une figure apprenante (transposition, vulgarisation), le passage des savoirs d'une langue à une autre (traduction, plurilinguisme). Notons que la dimension culturelle se pose aux deux niveaux, comme nous allons le voir.

Dans cette contribution, nous souhaitons nous arrêter de manière spécifique sur le processus d'alternance codique, bien décrit dans les études du biplurilinguisme, mais encore peu appliqué au cas des MOOC. L'alternance se présente à trois niveaux : macro (choix de langue pour telle capsule vidéo ou tel MOOC), 
micro (changement de langue plus ou moins spontané en cours d'énoncé) et méso (recours didactisé à deux ou plusieurs langues dans une même capsule ou leçon). La méso-alternance (Gajo et Steffen, 2015), dans le cas des $M O O C$, suscite un intérêt particulier et peut prendre des formes différentes, comme la citation dans une autre langue, l'interview bilingue, la projection de documents dans une langue différente de l'exposé. Mais la présence même des sous-titres illustre un cas de méso-alternance, dans la mesure où l'usager/ère bénéficie, en permanence, d'une langue à l'oral et d'une autre - ou de plusieurs autres à l'écrit.

Après avoir décliné de manière plus précise ces trois niveaux d'alternance, nous présenterons brièvement notre terrain de recherche et les trois MOOC - produits par l'Université de Genève et issus de différentes disciplines - sur lesquels porteront nos analyses. Nous nous pencherons alors sur les trois niveaux d'alternance codique en nous appuyant sur des extraits de $M O O C$, des déclarations des conceptrices et concepteurs, et des témoignages des usagers et usagères. Notre conclusion mettra en évidence la richesse des mécanismes d'alternance pour les $M O O C$ en lien avec une politique linguistique et éducative qui se place à l'articulation entre universalisation et contextualisation.

\section{Plurilinguisme, alternance des langues et médiation des savoirs dans les MOOC}

Le plurilinguisme peut se présenter de diverses manières dans le discours. On doit à Lüdi et Py (1986) la notion de marque transcodique (MTC), qui désigne la trace, dans un énoncé, de la présence de plus d'une langue. On pensera, notamment, aux interférences, aux emprunts et aux alternances codiques. Avant de nous pencher plus particulièrement sur ce dernier cas de figure, nous aimerions apporter deux réflexions générales quant au plurilinguisme (et aux MTC) dans le discours. Premièrement, il s'agit de distinguer entre les processus d'alternance (plurilinguisme pour travailler) et de contrastivité (plurilinguisme à travailler) (Gajo, 2019). Si l'alternance codique conduit à changer, en certains points du discours, la langue de communication ou d'exposition, la contrastivité convoque ponctuellement d'autres langues, à la manière d'une citation, à des fins d'explicitation ou de démonstration, par exemple. Deuxièmement, il est important de distinguer les différentes fonctions que jouent les MTC dans le discours. Ces fonctions se répartissent principalement selon deux perspectives : dans la perspective exolingue, les MTC servent avant tout à dépanner la communication suite à un problème linguistique (par exemple, on change de langue pour maintenir ou faciliter l'accès au contenu); dans la perspective bilingue, les MTC servent à marquer un élément du discours ou un paramètre contextuel (par exemple, on change de langue pour mettre en évidence une notion particulière ou parce que telle langue fait sens dans le contexte). La contrastivité relève de la perspective bilingue, alors que l'alternance peut relever, en fonction des cas, de l'une ou l'autre perspective. L'emprunt, quand il n'est pas fluide et intégré dans l'usage ordinaire, relève de la contrastivité. Dans le cas des MOOC, orientés vers une forme de vulgarisation, la question de l'usage ordinaire ne va pas de soi, car on trouve un « ordinaire du discours de spécialité » (entre expert-e-s) et un " ordinaire tout court ». Le fait de baliser le recours à un mot ou une expression issus d'une autre langue (par exemple, « comme on le dit en anglais », « en droit international, on utilise l'expression de... ») permet d'associer ces deux « ordinaires ». Penchons-nous maintenant de manière plus détaillée sur la question de l'alternance codique. 
L'alternance des langues est bien décrite dans les études du bi-plurilinguisme et notamment en lien avec le processus de construction des savoirs dans les interactions à caractère didactique (notamment Gajo et al., 2013). Elle intervient aux niveaux macro, méso et micro dans les enseignements (Duverger, 2009; Gajo, 2007).

Au niveau macro, lors de la conception et de la mise en place d'un enseignement, un choix de langues est effectué. Ce niveau macro est lié à un certain cadre et des contraintes institutionnelles, notamment une politique linguistique, dans lesquels s'inscrivent les enseignements. Dans le cas des MOOC, il s'agit de choisir une ou plusieurs langues pour les capsules vidéo et pour les traductions et le sous-titrage. Ces choix peuvent se porter sur des langues locales et nationales de l'institution où les $M O O C$ sont produits et dans lesquelles les savoirs à transmettre sont élaborés (langues des expertes et experts impliqués) et/ou des langues qui servent la diffusion internationale large (souvent l'anglais), mais ils peuvent aussi cibler des communautés d'étudiants et d'étudiantes issues de régions géopolitiques particulièrement concernées par la problématique abordée (langues des apprenantes et apprenants visés). Dans la perspective d'une transmission des savoirs et d'une accessibilité au niveau international et à large échelle, le choix de l'anglais apparait souvent comme un choix évident (voir Gajo et Steffen, 2014). Opter pour des langues dominantes et de confort pour le travail des expertes, experts, et/ou des apprenantes, apprenants est un choix plus orienté vers la médiation des savoirs. La question qu'on se pose dans ce cas est de savoir quelles langues sont susceptibles de servir l'appropriation des savoirs pour une thématique donnée dans un $M O O C$ particulier. Les savoirs médiatisés ne sont pas uniquement diffusés et mis à disposition, mais les acteurs et actrices impliqués peuvent agir dessus, qu'il s'agisse des formateurs, formatrices ou des apprenantes et apprenants (les savoirs sont traités et transformés dans la médiation numérique). Dans les deux cas, la traduction (sous-titrage plurilingue) des $M O O C$, de ou vers l'anglais et d'autres langues, sert la transmission massive des savoirs.

La méso-alternance se rapporte à un recours didactisé à deux ou plusieurs langues au sein d'un enseignement, lorsque l'enseignante ou l'enseignant prévoit de mettre en complémentarité et en contraste plusieurs langues comme outils de médiation et de construction des savoirs (Gajo et Steffen, 2015). Lors de la phase de didactisation, l'enseignante ou l'enseignant choisit notamment les contenus thématiques, les supports didactiques et les activités (définition, exemplification, synthèse, etc.) qu'il proposera aux apprenantes et apprenants, ainsi que la manière de les agencer (Steffen et Freytag Lauer, 2021). La mésoalternance se rapporte à l'alternance des langues qui est « raisonnée, réfléchie » (Duverger, 2007) à ce moment-là, en rapport à l'agencement des contenus et des activités et plus largement à la médiation des savoirs. Elle présente des traits communs avec le translanguaging, initialement décrit comme une pratique pédagogique selon laquelle on alterne les langues délibérément afin d'approfondir la compréhension du contenu (Williams, 1994, cité dans García et Wei, 2014). Plus récemment, les études s'intéressent au trans-semiotising, qui englobe toutes les ressources sémiotiques et multimodales exploitées dans un enseignement et non seulement les ressources plurilingues (par exemple Wu et Lin, 2019). Pour les $M O O C$, la méso-alternance peut prendre la forme d'une mise en contraste de la terminologie, qui vient mettre en mots et éclairer les savoirs sous différents angles dans différentes langues et dans les cultures et communautés scientifiques dans lesquelles elles s'implantent. Le choix, orienté vers l'apprenante ou l'apprenant, de proposer des supports visuels (schémas, mots-clés, etc. dans les capsules vidéo) ou écrits (de la lecture en complément des vidéos, par exemple) dans une ou des autres langues que celle utilisée dans les capsules vidéo constitue également une méso-alternance. Les sous-titres dans d'autres langues que celle de la vidéo représentent aussi une forme de méso-alternace, dans la mesure où l'apprenant ou l'apprenante dispose, pour la vidéo, d'un accès aux savoirs transmis à l'oral dans une langue et, pour les sous-titres, à l'écrit, dans une ou plusieurs autres langues. L'apprenant ou l'apprenante, dans son parcours 
d'apprentissage relatif à la thématique d'un $M O O C$, peut exploiter ces ressources plurisémiotiques', dépendant des langues proposées pour les sous-titres et de sa compétence dans ces langues. Dans le cas spécifique des $M O O C$, la méso-alternance ne se présente toutefois pas comme en classe. En effet, la personne qui apprend se trouve seule devant la leçon et décide de son parcours de manière très autonome. La méso-alternance peut alors l'aider ou l'encourager à emprunter un chemin plutôt qu'un autre - en raison de son accessibilité ou, au contraire, de sa nouveauté - et à opter, le cas échéant, pour un traitement largement monolingue et/ou monosémiotique.

La micro-alternance ou code-switching a été largement étudiée et se définit notamment comme l'alternance de langues qui se produit plus ou moins spontanément en cours d'énoncé. Dans le cadre des interactions en classe, elle est initiée par l'enseignante ou l'enseignant ou les apprenantes ou apprenants et a été analysée, notamment, en relation avec la construction des savoirs (Coste, 1997; Castellotti et Moore, 1997; Moore, 2001). Dans une interaction in vivo, la micro-alternance, définie en termes conversationnels, dépend des contingences locales et temporelles de celle-ci (voir Auer, 1988; Mondada, 2007), mais parlant du discours médiatisé dans une capsule vidéo d'un MOOC par exemple, la temporalité et la séquentialité du discours changent (possibilité de retour en arrière pour effacer et remplacer une séquence). II n'est donc pas sûr que nous rencontrions beaucoup de micro-alternances dans les MOOC, qui cèdent souvent la place aux emprunts. Par ailleurs, quand elles apparaissent, il est peu probable qu'elles se situent dans une perspective exolingue, qui vise à adapter la communication de l'expert ou experte à un apprenant ou une apprenante pour lui faciliter l'accès aux savoirs en cours de conversation (orientation vers la personne qui apprend), mais elles sont plutôt guidées par le choix de l'expert ou experte en fonction des savoirs à thématiser (orientation vers l'objet ou le sujet du discours).

\section{Présentation de la recherche et du corpus}

Les données que nous présenterons dans cet article émanent de la recherche "Plurilinguisme et construction des savoirs dans les MOOCs » (pour une présentation détaillée de notre étude, voir Fonseca et Gajo, 2020), dans laquelle nous nous intéressons à comprendre comment le recours au plurilinguisme dans les $M O O C$ peut amener des changements dans les pratiques éducatives aussi bien des concepteurs et conceptrices que des usagers et usagères. Pour ce faire, nous prenons en compte non seulement la perspective des formateurs et formatrices, et des apprenantes et apprenants, mais remettons également en question le caractère plus ou moins universel des savoirs transmis, dont la dimension culturelle devient particulièrement saillante lors du passage à une autre langue.

Le corpus que nous allons analyser dans cette contribution est composé de trois MOOC : « Drugs, drug use, drug policy and health », "Introduction aux droits de l'homme » et « Introduction au raisonnement clinique ». Ces cours ont été produits partiellement ou intégralement à l'Université de Genève et sont disponibles sur la plateforme Coursera ${ }^{2}$. Ces MOOC ont, en outre, différentes langues principales et de sous-titrage et appartiennent à divers champs disciplinaires (voir tableau 1), ce qui les rend particulièrement pertinents pour notre propos.

\footnotetext{
${ }^{1}$ Médiation des savoirs dans plusieurs langues et par différents modes sémiotiques (langue orale, langue écrite, illustration, carte, schéma etc.)

${ }^{2}$ https://www.coursera.org/
} 


\section{Tableau 1}

Aperçu du corpus analysé

\begin{tabular}{|c|c|c|c|}
\hline MOOC & $\begin{array}{l}\text { Champ } \\
\text { disciplinaire }\end{array}$ & $\begin{array}{l}\text { Langue } \\
\text { principale }\end{array}$ & $\begin{array}{l}\text { Langue } \\
\text { de sous-titrage }{ }^{3}\end{array}$ \\
\hline $\begin{array}{c}\text { Drugs, drug use, drug policy and } \\
\text { health }\end{array}$ & $\begin{array}{c}\text { Médecine/ } \\
\text { Sciences sociales }\end{array}$ & Anglais & $\begin{array}{c}\text { Français, Espagnol, } \\
\text { Russe, Italien et } \\
\text { Allemand }\end{array}$ \\
\hline $\begin{array}{l}\text { Introduction aux } \\
\text { droits de l'homme }\end{array}$ & Droit & Français & Anglais \\
\hline $\begin{array}{l}\text { Introduction au } \\
\text { raisonnement clinique }\end{array}$ & Médecine & Français & Anglais \\
\hline
\end{tabular}

Dans nos analyses, nous puiserons dans différents types de données, à savoir :

a) extraits d'entretiens semi-directifs menés avec les concepteurs et conceptrices;

b) témoignages des usagers/ères recueillis à travers des questionnaires de recherche intégrés aux MOOC étudiés, portant sur leur profil linguistique et leurs pratiques de visionnage en lien avec le sous-titrage;

c) extraits des $M O O C$ étudiés dans lesquels on observe des micro et méso-alternances ainsi que le sous-titrage de certains passages sélectionnés en une ou d'autres langues.

Nos analyses se basent sur des outils linguistiques issus de la perspective « plurilinguisme et cognition dans les pratiques discursives " (Gajo et al. 2013). Nous tentons ainsi de comprendre, à travers une analyse fine des marques linguistiques, les processus de transmission et de construction des savoirs en situation plurilingue. Nous avons notamment mis en évidence les liens entre les processus de médiation et de remédiation. Le premier concerne la nécessaire mise en discours des savoirs dans l'acte d'enseignement, qui s'appuie sur des ressources symboliques dont fait partie la langue choisie, mais aussi toutes les ressources propres à un domaine scientifique (tableaux, formules, cartes, etc.) et à un média (canal sonore, visuel, multicanalité, etc.). Le processus de remédiation, quant à lui, concerne avant tout le plurilinguisme, qui permet des entrées multiples - plusieurs médiations - sur les savoirs. Pour les enseignantes et enseignants, et les apprenantes et apprenants, l'outil plurilingue est d'abord placé au service de l'accessibilité et de la compréhension des savoirs (on écrirait alors "remédiation », car le recours à une autre langue permet de faire face à une difficulté), mais il peut aussi entrer dans une forme de problématisation des savoirs, questionnés par rapport au point de vue qu'ils proposent, supposent ou imposent. Ce double intérêt de la remédiation se retrouve dans un outil spécifique aux $M O O C$, celui du sous-titrage, largement documenté dans la présente recherche.

\footnotetext{
${ }^{3}$ Pour des raisons méthodologiques, nous avons fait le choix d'indiquer dans ce tableau seulement les langues de sous-titrage qui ont fait l'objet d'une demande de la part des concepteurs et conceptrices. Ces données ne prennent donc pas en compte le travail de sous-titrage effectué par la communauté des traducteurs et traductrices bénévoles de la plateforme Coursera.
} 


\section{Analyse des données}

\section{Macro-alternance}

Le choix opéré par les concepteurs et conceptrices - sélection de la ou des langues principales pour un MOOC - peut être décrit en termes de macro-alternance. De la même manière, le choix des langues de traduction et de sous-titrage, lorsqu'il y en a, relève également de la macro-alternance dans le sens où il est établi en amont. Comme indiqué plus haut, ce choix est souvent géopolitique, le sous-titrage servant à atteindre des usagers/usagères-apprenants situés dans des régions ciblées.

Du point de vue de leur structure, la plupart des $M O O C$ que nous avons analysés ont une seule langue/variété de référence, qui fonctionne comme la langue/variété principale de la plupart des vidéos. Ainsi, les 67 vidéos qui intègrent le $M O O C$ « Introduction aux droits de l'homme » sont majoritairement en français. Nous observons une structure semblable pour le $M O O C$ « Drugs, drug use, drug policy and health ", dont le parcours obligatoire comporte 55 vidéos en anglais et seulement 5 où d'autres langues sont majoritaires, notamment l'espagnol et le français. Dans certains $M O O C$, le choix de la langue principale n'intervient pas entre une langue et une autre, mais entre des variétés d'une même langue. Cela est le cas, par exemple, pour le MOOC "Introduction au raisonnement clinique ", cours élaboré par différentes universités francophones, dans lequel le français parlé en Suisse romande, et en particulier, à Genève, a été choisi comme la variété de référence.

On pourrait se demander pour quelle raison les concepteurs et conceptrices des $M O O C$ étudiés optent souvent pour une seule langue principale et une ou des langues de sous-titrage, selon les cas. Si une structuration autour d'une langue principale est la norme, il serait, néanmoins, possible d'imaginer une organisation dans laquelle les langues des capsules vidéo pourraient varier selon les sujets traités et les compétences linguistiques des intervenantes ou intervenants, amenant ainsi une grande polyphonie dans le MOOC. Dans la plupart des $M O O C$ analysés, le recours à une seule langue principale pour la transmission des savoirs semble s'expliquer par trois raisons: la représentation sur les langues que peuvent avoir les concepteurs et conceptrices, l'importance de certaines langues pour la discipline en question ainsi que les contraintes imposées par les plateformes où les MOOC sont diffusés (voir Fonseca et Gajo, 2021a). Si le plurilinguisme est possible dans les MOOC, la plupart du temps, le choix s'oriente vers une seule langue. A l'Université de Genève, il s'agit soit de l'anglais, soit du français'.

Du point de vue des représentations sociales, pour certaines disciplines, comme les sciences médicales, s'exprimer dans une autre langue que l'anglais pourrait même mettre en question la légitimité des propos, comme on l'observe dans le passage ci-dessous, extrait d'un entretien avec les concepteurs et conceptrices du $M O O C$ « Drugs, drug use, drug policy and health » :

moi ce qui m'intéresse surtout c'est effectivement [...] quand voilà la personne présente et où tu parles anglais est-ce que c'est considéré comme plus scientifique par l'apprenant que le français/ [...] parce que l'anglais est [une] langue disons scientifique [...] est-ce que ça a une autre valeur si quelqu'un s'exprime en français/ en espagnol ou autre chose [...]

\footnotetext{
${ }^{4}$ Ainsi, en décembre 2020, parmi les 43 MOOC produits par cet établissement, 25 étaient en anglais et 18 en français. Parmi les $M O O C$ en anglais, 5 ont été sous-titrés vers d'autres langues à la demande des concepteurs et conceptrices, ce nombre s'élevant à 8 pour les $M O O C$ en français.
} 
Si l'on comprend la préoccupation du concepteur ou de la conceptrice, étant donné l'importance de l'anglais pour les sciences médicales, plusieurs témoignages des usagers et usagères recueillis dans le cadre de notre recherche nous rappellent que le recours massif à une seule langue dans la diffusion des connaissances peut finir par appauvrir les propos des intervenantes ou intervenants, notamment lorsque ceux-ci ne maitrisent pas suffisamment la langue en question. C'est ce que nous rappellent les propos cidessous d'un usager ou usagère-apprenant ou apprenante ayant suivi ce MOOC :

Subtitles are important tools to enable broader access to the course. They may also help understanding speakers whose English pronunciation is not very good (in such cases, it is best that they speak French, or whatever other language they speak best) (ID 90).

Contrairement à la représentation courante, recourir à une seule langue, le plus souvent l'anglais « lingua academica » (voir plus bas), ne semble pas une solution pour la transmission des savoirs dans les MOOC. L'argument concerne toutefois ici essentiellement la qualité de la langue de l'intervenante ou de l'intervenant, et beaucoup moins l'intérêt du plurilinguisme en tant que tel, qui ressort davantage au niveau de la méso-alternance.

Le recours réfléchi au plurilinguisme, à différents niveaux, entre des capsules vidéo (langues de référence différentes selon les capsules), ou alors à l'intérieur d'une même capsule (à travers, par exemple, le recours à des citations, interventions dans d'autres langues), rendu possible grâce au sous-titrage, peut constituer une vraie plus-value pour la construction des savoirs et mériterait d'être exploité dans le cadre des MOOC. Dans la section suivante, nous allons observer comment l'alternance des langues peut être utilisée à des fins didactiques, en nous basant tout d'abord sur la perspective des concepteurs et conceptrices, et ensuite sur celle des usagers et usagères-apprenants.

\section{Méso-alternance et multimodalité}

Dans les $M O O C$ étudiés, on observe davantage le potentiel didactique que peut représenter le recours à la méso-alternance plutôt que son exploitation effective par les concepteurs et conceptrices. Dans le MOOC "Introduction aux droits de l'homme », par exemple, la méso-alternance marque souvent le passage d'un discours en français, langue principale, à l'anglais, langue qui intervient à travers la lecture d'une citation également projetée en arrière-plan. Dans un tel cas, le changement de langue n'agit pas tant en termes de problématisation, mais de polyphonie, contribuant à rendre audibles d'autres voix dans le discours de l'enseignante ou de l'enseignant. C'est cela que l'on remarque dans les images ci-dessous, qui reprennent, respectivement, l'annonce d'une citation formulée dans un écrit oralisé (« voici un passage clé ») (figure 1), la citation à proprement parler (lue et projetée en anglais) (figure 2) ainsi que la reprise du discours en français, immédiatement après la lecture de la citation (figure 3 ). 


\section{Figure 1}

Annonce d'une citation - MOOC "Introduction aux droits de l'homme », semaine 2, vidéo "La naissance des droits de l'homme »

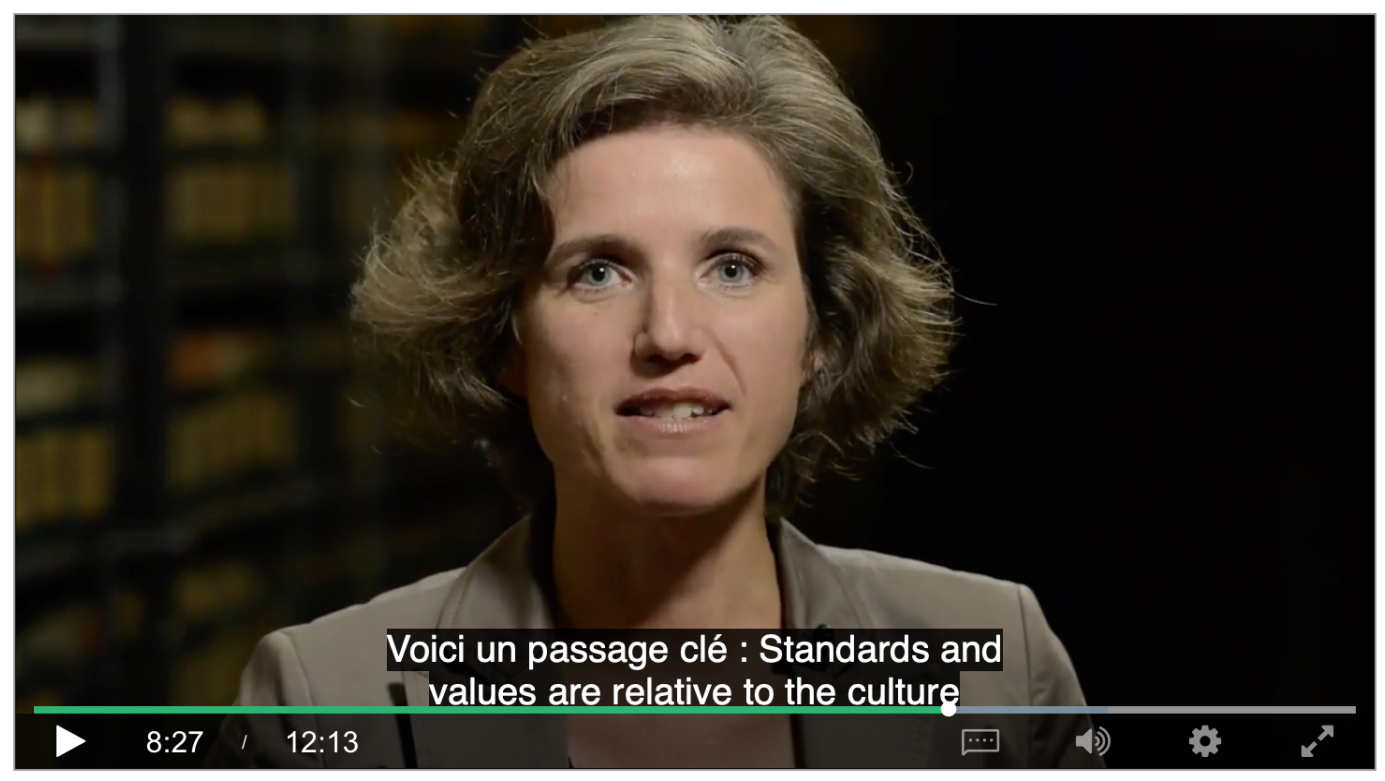




\section{Figure 2}

Citation lue et projetée en anglais - MOOC «Introduction aux droits de l'homme », semaine 2, vidéo "La naissance des droits de l'homme "

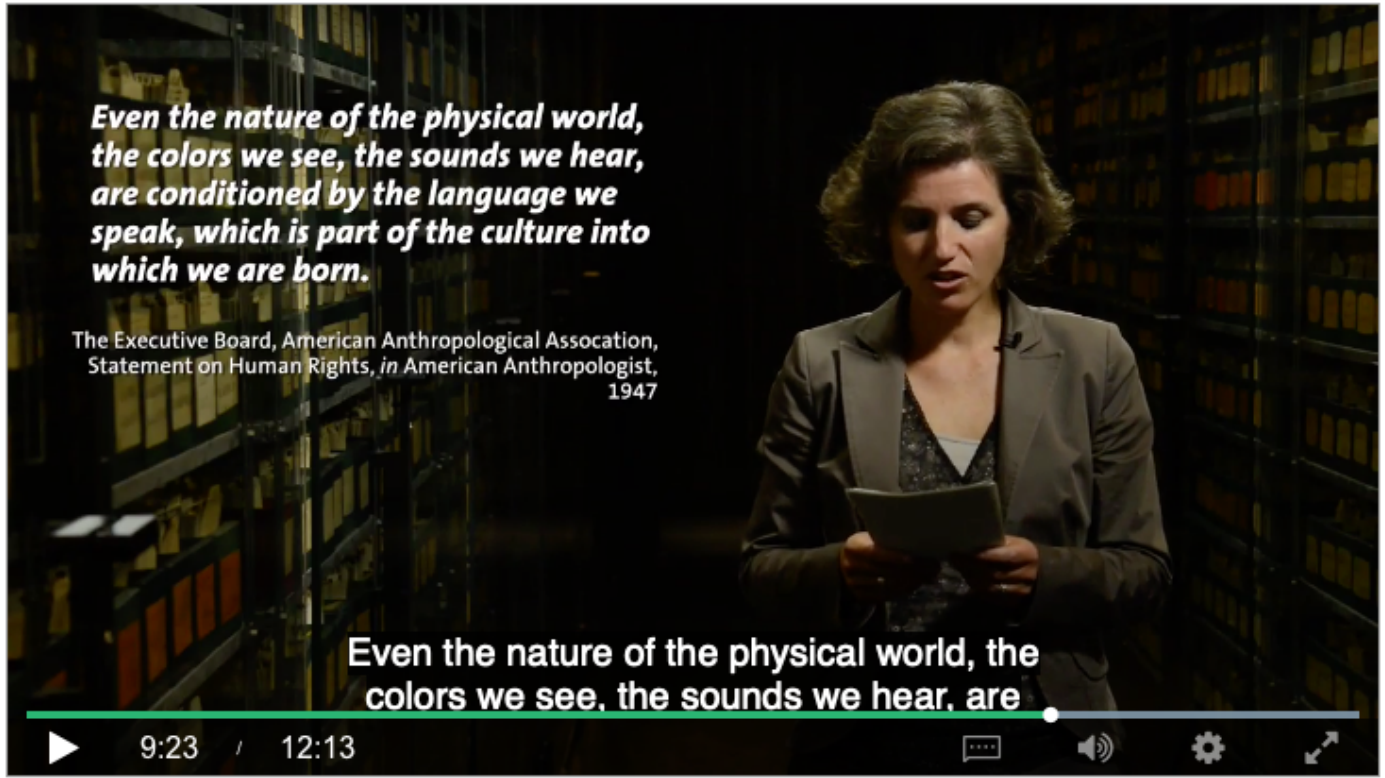

\section{Figure 3}

Reprise du discours en français après la lecture de la citation - MOOC "Introduction aux droits de l'homme », semaine 2, vidéo "La naissance des droits de l'homme »

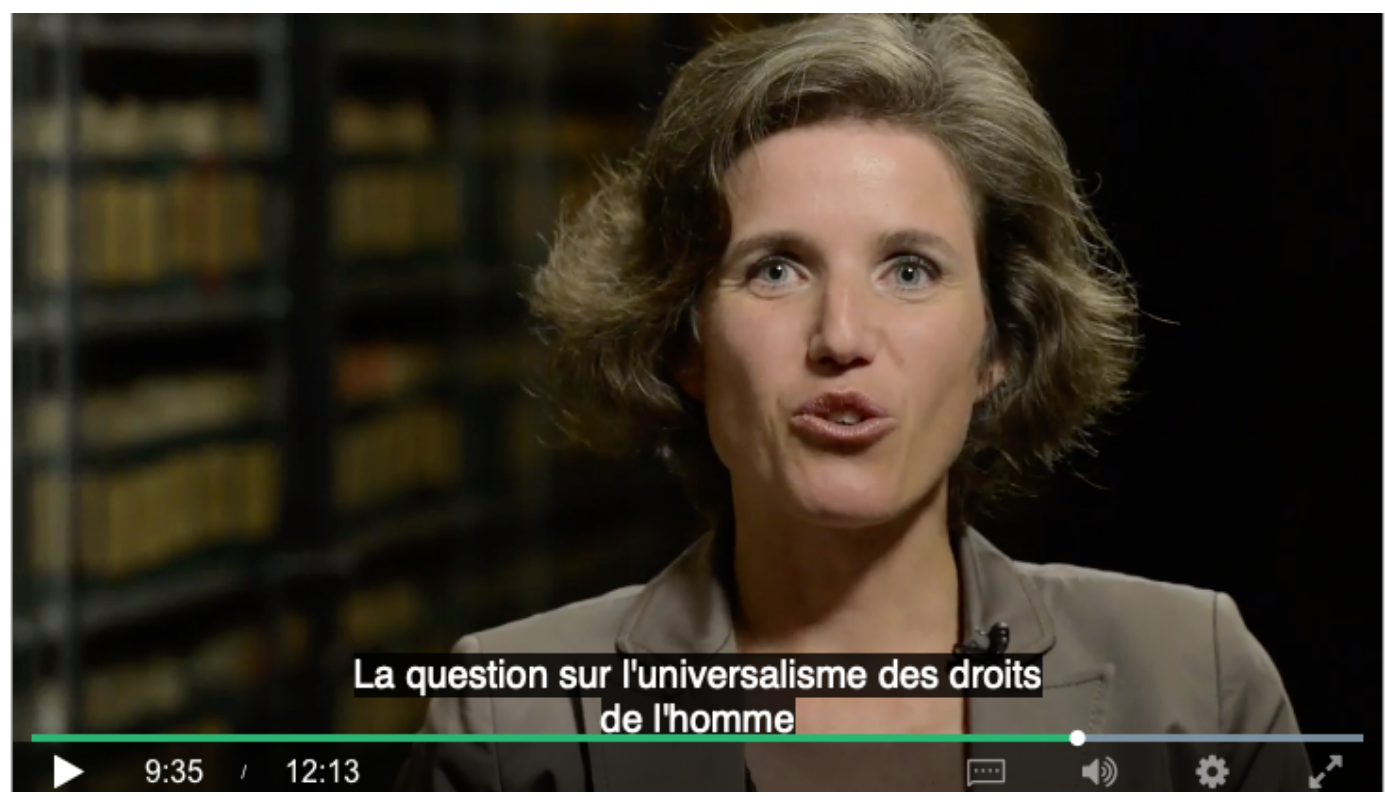


Dans le $M O O C$ « Drugs, drug use, drug policy and health », la méso-alternance est présente sous une autre forme. Elle intervient notamment lors d'entretiens, en particulier à l'occasion du passage de parole entre l'interviewer ou intervieweuse à la personne interviewée. C'est ce que l'on observe dans les images ci-dessous où l'intervieweuse introduit le sujet de la vidéo en anglais, langue principale du MOOC (figure 4), et s'adresse ensuite à l'interviewée en espagnol, langue dans laquelle cette dernière développera ses propos (figure 5). Si le changement de langue a ici une fonction plus sociale que didactique (saluer l'intervenante et lui poser une question dans sa langue), il contribue, comme dans l'exemple précédent, à rendre le $M O O C$ polyphonique, permettant aux usagers/ères-apprenant-es d'avoir accès à la parole d'intervenantes et intervenants situés dans différents espaces et parlant plusieurs langues.

\section{Figure 4}

Introduction du sujet de la vidéo en anglais - MOOC "Drugs, drug use, drug policy and health", semaine 4, vidéo "Violations of human rights in Central America and South America »

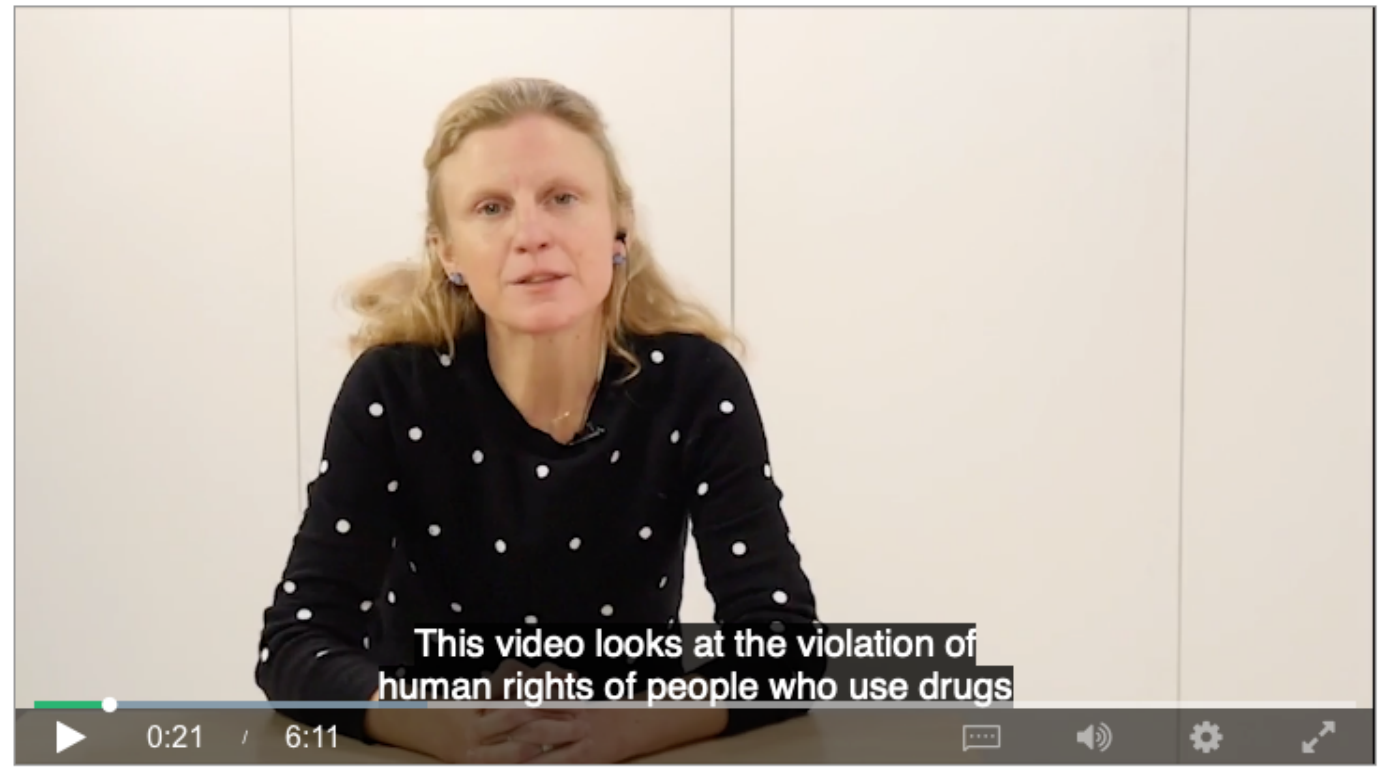




\section{Figure 5}

Question posée en espagnol, langue de l'interviewée - MOOC " Drugs, drug use, drug policy and health », semaine 4, vidéo "Violations of human rights in Central America and South America »

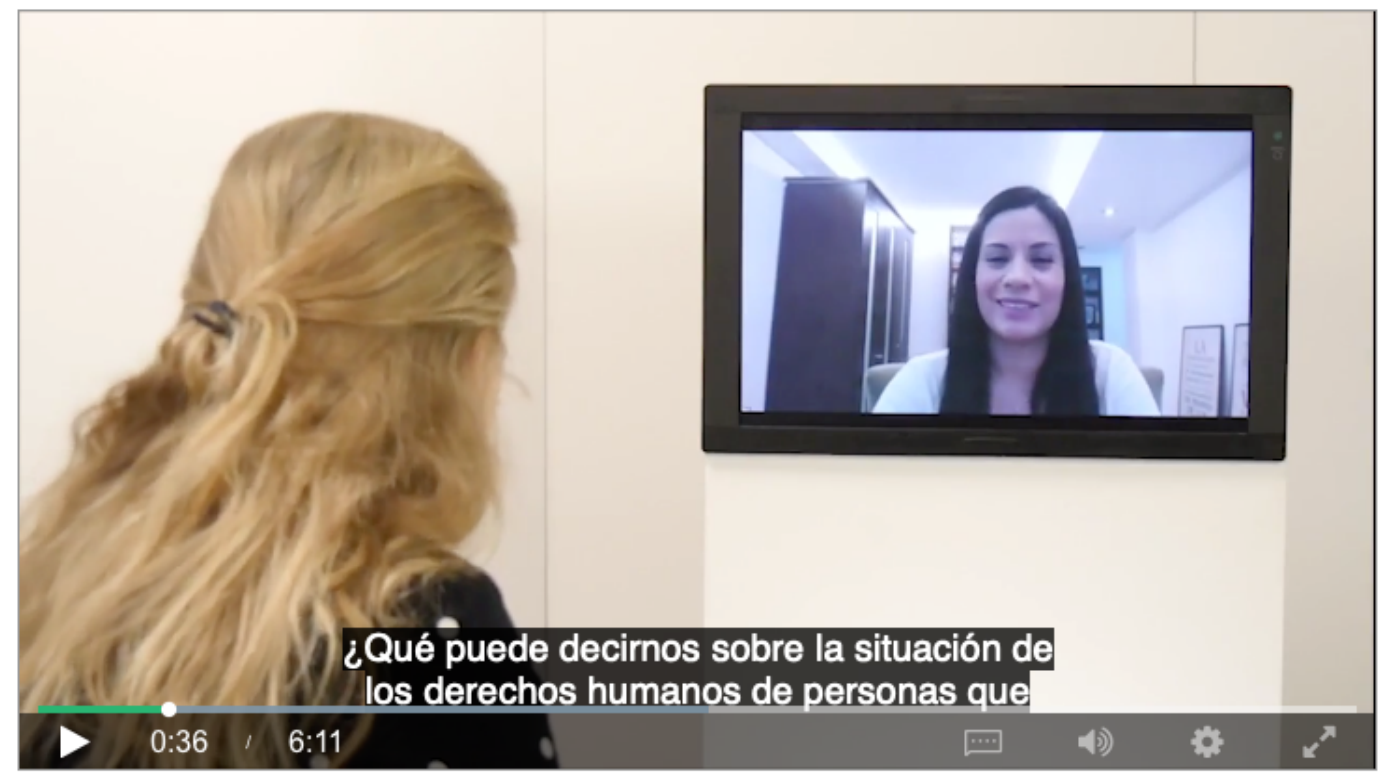

Malgré les formats différents dans lesquels la méso-alternance intervient (citation, interview), ces exemples se ressemblent par la façon dont les langues sont agencées : les langues sont juxtaposées à l'intérieur d'une même séquence vidéo sans qu'il y ait de questionnement explicite sur ce que l'autre langue convoquée pourrait apporter pour la compréhension de la thématique traitée. Le recours à la mésoalternance vise ainsi plus la communication que la problématisation. Cela dit, le changement de langue renforce aussi le processus de contextualisation (l'espagnol pour parler d'une réalité latino-américaine, par exemple) des questions traitées et donne sans doute un caractère plus crédible au discours. Cette contextualisation intervient toutefois plus au niveau des données de terrain que des analyses proposées, d'où son lien avec le format « témoignage ».

Cette même visée - accès plus aisé à l'information dans une $L 2^{5}$ - est celle qui se retrouve le plus souvent du côté des usagers/ères-apprenant-es qui visionnent un $M O O C$ sous-titré dans une autre langue. Comme exposé plus haut, le sous-titrage plurilingue constitue une forme de méso-alternance dans le sens où deux langues sont coprésentes à travers des canaux et des modes sémiotiques différents : à l'oral, à l'écrit, dans les sous-titres, les citations ou schémas. Si jusque-là la méso-alternance a été décrite par rapport à des interactions didactiques in vivo, son analyse dans des discours médiatisés à caractère didactique met en évidence d'autant mieux son rapport à la multimodalité et aux ressources didactiques plurisémiotiques (plusieurs modes et canaux sémiotiques) et l'importance de rendre compte du processus de construction et de transmission des savoirs dans sa globalité. La multicanalité et la plurisémioticité remettent aussi en question la séquentialité de la méso-alternance. En effet, si cette dernière constitue une ressource mise à disposition par les concepteurs et conceptrices, elle n'empêche pas forcément des parcours monolingues

\footnotetext{
${ }^{5}$ L2 est utilisée pour « deuxième langue ».
} 
et monosémiotiques. L'accès à l'information peut ainsi être renforcé par le retour en arrière possible (répétition de la séquence) autant sinon plus que par le plurilinguisme et la multimodalité. Encore une fois, cela concernerait davantage la communication des contenus que la problématisation des savoirs.

Dans une des vidéos introductives du MOOC « Drugs, drug use, drug policy and health ", l'intervenant s'attèle à définir le terme anglais "drugs » (figure 6, "What do we mean by drugs », 0:30) en partant du constat que ce terme est polysémique (figure 6 , « the term drug is polysemous », 0:33). Il explicite ensuite les différents sens du terme (sens générique, sens pharmacologique, sens commun, etc.) pour terminer avec la définition scientifique adoptée dans le $M O O C$, basée sur la polysémie bienvenue du terme anglais " drug » utilisé couramment pour signifier à la fois « drogue » et « médicament » (cela n'est le cas ni pour le terme français « drogue », ni pour le terme allemand « Droge » notamment). Pour les usagers, usagèresapprenants de ce $M O O C$, il peut, par conséquent, s'avérer intéressant, pour sa compréhension des différentes nuances du terme - introduites à ce moment-là, puis utilisées et approfondies tout au long du MOOC - de pouvoir consulter en parallèle les sous-titres dans d'autres langues, d'accéder à la terminologie utilisée dans d'autres langues et qui découpent et mettent en mots les différents aspects de ce concept de manière variable. La méso-alternance s'opère entre l'oral de la vidéo en anglais (figure 6), la définition écrite en anglais insérée et commentée dans la vidéo (figure 7) et l'écrit des sous-titres dans une ou plusieurs autres langues (figures 6 et 7 ). 
Définition à l'oral en anglais avec sous-titres dans une autre langue - MOOC "Drugs, drug use, drug policy and health », semaine 1, vidéo "Introduction to module 1 », 0.30-2:16

What do we mean by drugs? The term drug is polysemous. [...] In the broadest sense, a drug is any substance, medicine or poison that has biochemical effects on either mind or body. However, for substances that act on the mind, psychoactive substances, [...] the term drugs has acquired a negative meaning. In the pharmacological sense, caffeine, nicotine, and alcohol are drugs just as cocaine and heroin are. In popular usage, drug has taken on a different meaning. Over the last century, drug has come to mean a psychoactive substance that is illegal. [...] In this $M O O C$, we will assume the ambiguous meaning of the word drug in English, which perfectly reflects the fact that psychoactive substances can be both essential medications and potentially addictive and dangerous, both beneficial and harmful.

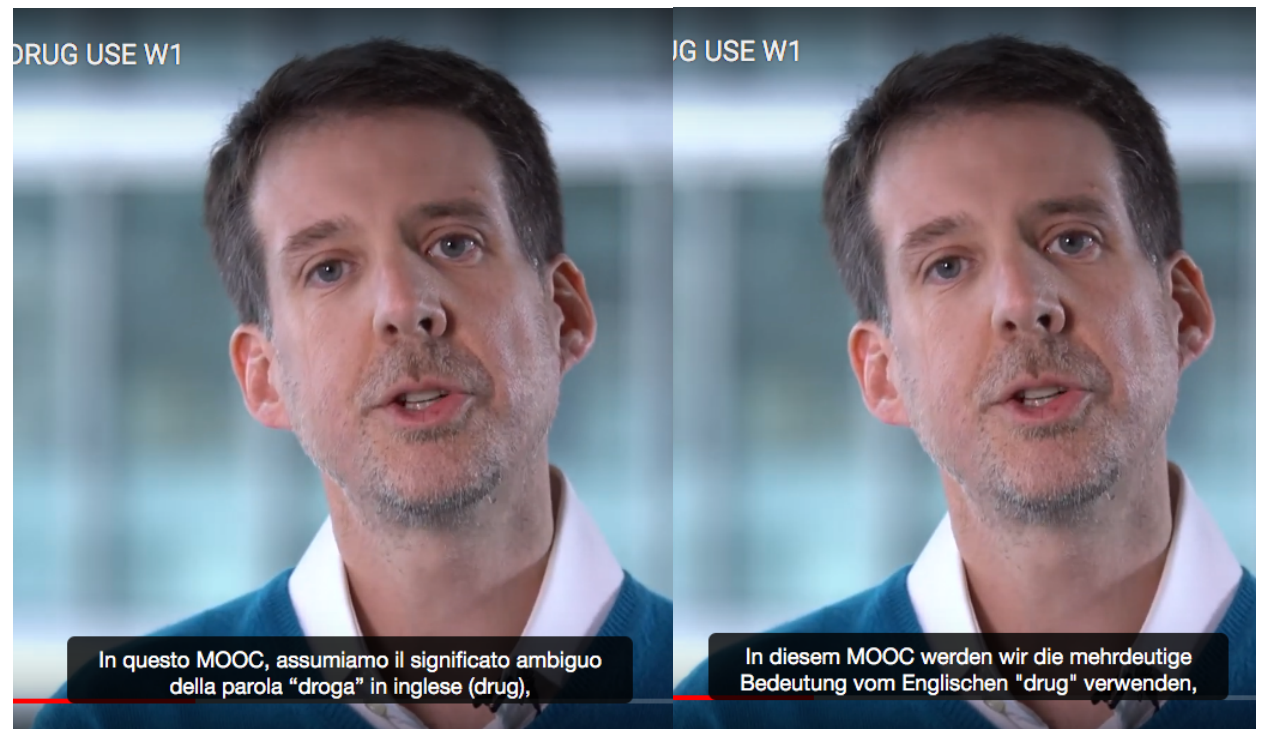




\section{Figure 7}

Définition à l'écrit en anglais avec sous-titrage dans une autre langue - MOOC « Drugs, drug use, drug policy and health », semaine 1, vidéo "Introduction to module 1 »

Global Commission on Drug Policy report The World Drug Perception Problem, 2017 : "In the broadest sense, a drug is any substance - medicine or poison - that has a biochemical effect on either mind or body. However, for substances that act on the mind (psychoactive substances), including stimulants, sedatives, psychedelics, hypnotics, hallucinogenics or dissociatives, the term drug has acquired a negative meaning. In the pharmacological sense, caffeine, nicotine and REVICoshol are drugs just as cocaine and heroin are.

alcohol are drugs just as cocaine and heroin are.
"Im weitesten Sinne ist eine Droge jede
Substanz - Arzneimittel oder Gift -

In popular usage, 'drug' has taken on a different meaning. Over the last century, 'drug' has come to mean a psychoactive substance that is illegal. In this sense, cannabis is a drug while alcohol is not (in most countries at any rate); and substances such as morphine are "medicines" when used by doctors, and "drugs" when used recreationally.

Drugs are more socially accepted when supplied as medicines. Whether a substance is a drug in this usage depends on the intention behind its use, the mode of administration and the social class of the user; and while in many cases the active substances remain the same the perception is very distinct."

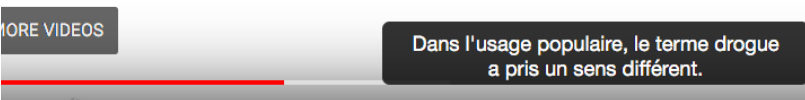

Dans nos travaux, nous avons documenté, sur la base de questionnaires, les pratiques des usagers/èresapprenant-es en lien avec le sous-titrage et les fonctions qui en ressortent (Fonseca et Gajo, 2020). D'une manière générale, nos données montrent que si la plupart des usagères et usagers-apprenants affirment que le sous-titrage plurilingue leur permet de mieux comprendre ce qui est dit, certains indiquent qu'il leur donne la possibilité d'apprendre une autre langue et d'autres, minoritaires, rapportent qu'il leur sert à comparer des informations dans différentes langues (Fonseca et Gajo, 2021b). Cette dernière catégorie fait état d'une exploitation active des langues et des ressources plurilingues pour le questionnement des savoirs. 
Les témoignages que nous présenterons ci-dessous, extraits des questionnaires de recherche proposés aux usagers et usagères-apprenants du MOOC « Introduction aux droits de l'homme », montrent que les sous-titres bénéficient non seulement à la compréhension des savoirs étudiés à travers la comparaison des informations fournies dans la langue principale du MOOC et, respectivement, dans sa traduction et le sous-titrage dans une autre langue (premier extrait), mais qu'ils peuvent également permettre un double apprentissage, à la fois linguistique et disciplinaire (second extrait).

[Les sous-titres] me permettent de comparer les informations, de regarder la traduction des motsclés et les concepts (ID 755).

[los subtítulos me permiten] entender y comprender el idioma extranjero al mismo tiempo que poder compararlo con el idioma nativo sobre un tema determinado como el derecho en este caso (ID 771).

La comparaison des langues et des savoirs médiatisés par différentes langues dont témoignent les usagers/ères-apprenant-es est rendue possible par les caractéristiques du dispositif $M O O C$, qui, à travers le sous-titrage plurilingue, permet une forme de méso-alternance. Observons, par ailleurs, que la présence simultanée de deux langues à travers deux canaux différents (oral et écrit), propre à cette forme de mésoalternance, est mise en avant par le second commentaire, à travers la formulation " en même temps " (" al mismo tiempo »). II reste difficile de savoir, toutefois, si cette dernière expression renvoie, de manière générale, au double objectif que l'on peut poursuivre en consultant le $M O O C$ ou, plus précisément, à un mode de travail impliquant la séquentialité. Si la multicanalité et le plurilinguisme sont exploités didactiquement par certains usagers et usagères-apprenants, il reste à la transformer en une réelle plusvalue pour les concepteurs et conceptrices, ce qui enrichirait les savoirs transmis et construits à travers les MOOC.

\section{Micro-alternance et contrastivité}

Si la micro-alternance intervient dans les MOOC, ses occurrences sont relativement limitées et ses fonctions peu diversifiées. Cela tient au caractère largement préformaté de ce type d'enseignement, qui s'éloigne considérablement d'une conversation à bâtons rompus. Par ailleurs, le « problème » que pourrait représenter le plurilinguisme y est pensé en amont, ce qui contribue à "éviter " un certain nombre de marques transcodiques (MTC). Ce caractère programmé de l'interaction plurilingue débouche toutefois sur un trait intéressant, à savoir le fait que les MTC, quand elles se produisent, ne relèvent pas tant d'une dimension exolingue que d'une dimension bilingue. Plus précisément, cela signifie que le passage d'une langue à l'autre, par exemple, est utilisé pour produire un effet particulier (fonction rhétorique, enrichissement cognitif, etc.) et non pas pour débrouiller un problème de communication.

[...] le travail ne doit pas être considéré simplement comme marchandise ou comme un article de commerce. $\mathrm{Ou}$, pour le dire de façon plus succincte en anglais : labor is not a commodity $(\mathrm{DH}: \mathrm{W} 2 \mathrm{~V} 3,8.28-8.40)$.

Dans cet extrait comme dans d'autres, on remarque tout d'abord que la micro-alternance est non seulement balisée (marquée, annoncée), mais justifiée. Le passage à l'anglais est donc recherché pour une raison particulière, ici parce qu'il permet de formuler l'argument de manière plus succincte. II en va non seulement d'une forme d'efficacité communicative, mais aussi d'une probable plus-value cognitive. En effet, "labor is not a commodity » représente une formulation plus synthétique - et, par-là, plus abstraite et conceptualisante - que la version française, qui la précède utilement. Les deux langues se 
complètent ainsi et contribuent, ensemble, à la construction et à la transmission des savoirs. Ce potentiel programmé de la micro-alternance est rendu possible par le plurilinguisme des concepteurs et conceptrices, qui traversent leur discipline dans une diversité de langues et en mesurent sans doute l'intérêt.

Ce caractère programmé de l'alternance, quand il rencontre des objectifs didactiques, rapproche les enjeux micro et des enjeux méso. La didactisation du plurilinguisme dans les MOOC, au niveau micro, se traduit toutefois plus par des phénomènes de contrastivité que par des phénomènes d'alternance, c'est-àdire que l'on cite d'autres langues, on s'y réfère sans pour autant changer la langue de communication.

On ne parle pas de droits de l'homme, mais plutôt de droits fondamentaux, de droits constitutionnels, de liberté publique, ou, dans les pays anglosaxons, de civil rights, ou civil liberties (DH : W1V2, 9.38 - 9.50).

Dans cet extrait, on thématise à plusieurs reprises la désignation des droits de l'homme, avec ses variations en français déjà (" droits de l'homme » vs "droits humains ») et les reformulations proposées ici (« droits fondamentaux », " droits constitutionnels »). La référence à d'autres langues offre toutefois des angles de réflexion intéressants. Cette référence est amenée ici sur la base d'un ancrage plus culturel (" dans les pays anglo-saxons ») que linguistique. Ceci met en évidence la tension entre contextualisation et universalisation qui anime les MOOC. En effet, si ces derniers s'adressent au plus grand nombre en se servant le plus souvent d'une langue principale de communication, ils gagnent à ne pas écraser le rôle de la diversité linguistique et culturelle à l'œuvre dans la fabrication des savoirs savants. La mise en contraste entre différentes langues contribue ainsi à montrer une diversité de points de vue, d'autant plus importante dans des disciplines relevant des sciences humaines et sociales.

Un enseignant ou une enseignante de droit, de surcroit plurilingue, se montre en général sensible à ces questions. Les entretiens conduits avec les concepteurs et conceptrices de MOOC, tout comme les corrections apportées par ces derniers/ères à la traduction proposée par les sous-titres, confirment cette attention à la formulation et à la non-superposition des différentes langues (voir Fonseca et Gajo, 2021a). À propos de la notion de "droit » en lien avec les "droits de l'homme ", une des conceptrices commente de la manière suivante la correction apportée à la traduction anglaise : " J'ai fait cet ajout [individual entitlements ou lieu de "Human Rights are Rights"], car en français, l'élément définitionnel (droits subjectifs) apporte quelque chose, alors qu'en anglais "Human rights are rights" est tautologique ».

L'exposition de cette diversité d'ancrages et de points de vue dans un MOOC renforce, paradoxalement, leur ambition universelle, dans la mesure où le socle des fondements scientifiques, pour être accepté et partagé à large échelle, doit reposer sur une prise en compte de cette diversité. Une telle prise en compte peut déboucher sur diverses options :

- Adopter une sorte de dénominateur commun aux différents points de vue;

- Assumer un point de vue (une version de la connaissance) tout en ne discréditant pas l'existence et la légitimité d'autres points de vue;

- Construire un discours original à partir de cette diversité.

Cette ambition universelle peut passer par la mise en contraste de langues et de cultures a priori fort éloignées, comme le français et le chinois : "Elle visait à codifier la vertu centrale du confucianisme dite "ren". "Ren" implique tout d'abord la conscience qu'il y a d'autres hommes. "Ren" implique également le 
respect pour autrui » (DH : W1V3, 4.15 - 4.29). Le chinois, utilisé ponctuellement, peut donc contribuer à cette universalité, d'une manière certes différente de l'anglais, plus régulièrement présent. Même en sciences humaines et sociales, l'anglais fonctionne souvent comme « lingua academica » (voir Berthoud et Gajo, 2020), d'autant plus dans un domaine à forte couverture internationale, comme les droits de l'homme : «On a aussi vu apparaitre des Legal Black Holes : des régimes à part [...] 》 (DH : W1V3, $7.43-7.49$ ). Ainsi, un peu à la manière de l'informatique, on produit des notions souvent d'abord en anglais, et elles circulent ensuite largement sans être forcément adaptées ou traduites. Le fait de se référer à la désignation anglaise permet aussi, dans certains cas, d'activer de manière plus immédiate la dimension internationale.

\section{Conclusion}

Le sous-titrage des $M O O C$ relève, dans un premier temps, d'une volonté plutôt pratique au service d'une communication large. Sur la plateforme Coursera, il est d'ailleurs proposé par des bénévoles. Interroger le sous-titrage à travers le regard des concepteurs et conceptrices, des usagers et usagères, et des linguistes permet d'ouvrir une large réflexion sur les diverses formes et fonctions du plurilinguisme dans les MOOC. Très souvent, on adopte, par défaut, les modalités OLON (« one language only ») ou OLAT ( " one language at a time »), alors qu'il est possible de tirer un véritable bénéfice des modalités ALAST (" all the languages at the same time ») ou ALAT (« all the languages at all time ») (voir Lüdi, Höchle et Yanaprasart, 2016). Ceci concerne avant tout le niveau macro, qui distribue en amont les langues de travail. Une prise de conscience à ce niveau-là permet ensuite d'exploiter de manière plus riche la diversité des langues aux niveaux méso et micro.

Une telle richesse existe déjà dans les $M O O C$ que nous avons analysés, mais elle tient souvent au plurilinguisme des concepteurs et conceptrices eux-mêmes qui, même quand ils interviennent majoritairement dans une langue, arrivent à problématiser des éléments de savoir en lien avec le plurilinguisme (ceci touche essentiellement le niveau micro et, en particulier, le processus de contrastivité).

Le plurilinguisme dans les MOOC reconsidère, par ailleurs, la question de la contextualisation, en constante tension avec celle de l'universalisation. Comme nous l'avons vu, la contextualisation peut affecter, d'une part, les données de terrain et, de l'autre, les savoirs eux-mêmes. Si la contrastivité agit sur ces derniers, la méso-alternance, telle qu'exploitée par les concepteurs et conceptrices, intervient, elle, principalement en lien avec les données/réalités de terrain, à travers des témoignages et des illustrations. Exploiter la méso-alternance au service des savoirs ouvrirait alors un important espace de développement pour la mise en place de futurs $M O O C$ sensibles à la question du plurilinguisme et, plus généralement, des discours et des langues dans la transmission des savoirs. II s'agirait, pour cela, de mettre la polyphonie au service de la problématisation des savoirs. La question des genres textuels, des formats pédagogiques peut elle aussi être accrochée à des enjeux de plurilinguisme et de contextualisation. Cette question s'attarde, à sa manière, sur les enjeux culturels des savoirs et de leurs modes de transmission.

Dans cet article, nous ne nous sommes pas arrêtés sur les liens entre alternance codique et contraintes techniques imposées par le sous-titrage (médiatisation), qui limite la place des incrustations à l'écran et entraine parfois un raccourcissement des propos et, çà et là, une forme de simplification ou alors une clarification (médiation). La dynamique entre médiatisation, médiation et plurilinguisme dans les MOOC mérite encore une attention particulière de la part de la recherche. 


\section{Liste de références}

Auer, P. (1988). A Conversation Analytic Approach to Code-Switching and Transfer. Dans M. Heller (dir.), Codeswitching: anthropological and sociolinguistic perspectives. Berlin : Mouton de Gruyter. 187-213.

Berthoud, A.-C. et Gajo, L. (2020). The Multilingual Challenge for the Construction and Transmission of Scientific Knowledge. John Benjamins, Prestige Dylan Series.

Castellotti, V. et Moore, D. (1997). Alterner pour apprendre, alterner pour enseigner. Études de Linguistique Appliquée, 108, 389-392.

Cavalli, M. et Coste, D. (2019). Décrire l'activité langagière de médiation : le cas de l'enseignement bilingue. Cahiers de l'Asdifle, 30, 67-93.

Coste, D. (1997). Alternances didactiques. Études de linguistique appliquée, 108, 393-400.

Duverger, J. (2007). Didactiser l'alternance des langues en cours de DNL. Tréma, 28, 81-88. https://doi.org/10.4000/trema.302

Duverger, J. (2009). Favoriser l'alternance des langues. Le Français dans le monde, 362, 26-28. https://www.fdlm.org/blog/2010/06/29/bonjour-tout-le-monde-2/

Fonseca, M. et Gajo, L. (2020). Le plurilinguisme dans les MOOCs: Profils d'usagers et fonctions du sous-titrage. Alsic. Apprentissage des Langues et Systèmes d'Information et de Communication, 23(2). https://doi.org/10.4000/alsic.4816

Fonseca, M. et Gajo, L. (2021a). Le plurilinguisme dans les MOOCs : de nouvelles ressources pour la construction des savoirs. Bulletin suisse de linguistique appliquée [n spécial], 2, 165-181.

Fonseca, M. et Gajo, L. (2021b). Le sous-titrage plurilingue dans les MOOCs : une ressource pour l'apprentissage des langues? MOOCs, language learning and mobility, design, integration, reuse. Colloque en ligne, Italie. https://hal.archives-ouvertes.fr/hal-03228215

Gajo, L. (2007). Enseignement d'une DNL en langue étrangère : de la clarification à la conceptualisation. Tréma, 28, 37-48. http://doi.org/10.4000/trema.448

Gajo, L. (2019). Contexte plurilingue, dispositif bi-plurilingue, modalité bi-plurilingue d'enseignement: quelques repères. Cahiers de l'ASDIFLE, 30, 95-111.

Gajo, L., Grobet, A., Serra, C., Steffen, G., Müller, G. et Berthoud, A.-C. (2013). Plurilingualisms and knowledge construction in higher education. Dans A.-C. Berthoud, F. Grin et G. Lüdi (dir.), Exploring the Dynamics of Multilingualism: The DYLAN project. Amsterdam : John Benjamins. 287-308. https://doi.org/10.1075/mdm.2.13gaj

Gajo, L. et Steffen, G. (2014). Science et plurilinguisme : savoirs et perspectives en tension. Dans A.-C. Berthoud et M. Burger (dir.). Repenser le rôle des pratiques langagières dans la constitution des espaces sociaux contemporains. Champs linguistiques Recueils. Paris, Bruxelles : DeBoeck. 107-124.

Gajo, L. et Steffen, G. (2015). Didactique du plurilinguisme et alternance de codes : le cas de l'enseignement bilingue précoce. Revue Canadienne des Langues Vivantes, 71(4), 471-499. https://doi.org/10.3138/cmlr.2740

García, O. et Li Wei. (2014). Translanguaging: Language bilingualism and Education. Basingstoke : Palgrave Macmillan.

Lüdi, G. et Py, B. (1986). Être bilingue. Berne: Peter Lang.

Lüdi, G., Höchle, K. et Yanaprasart, P. (dir.). (2016). Managing Plurilingual and Intercultural Practices in the Workplace. John Benjamins.

Mondada, L. (2007). Le code-switching comme ressource pour l'organisation de la parole-en-interaction. Journal of Language Contact, 1, 168-197. https://hal.archives-ouvertes.fr/halshs-00356554

Moore, D. (2001). Une didactique de l'alternance pour mieux apprendre ? Études de Linguistique Appliquée, 121, 71-78. https://doi.org/10.3917/ela.121.0071

Steffen, G. et Freytag Lauer, A. (2021). Ilots bilingues et méso-alternance : une approche plurilingue des DNL. Recherches en didactique des langues et des cultures [en ligne], 18-3. https://doi.org/10.4000/rdlc.9853

Wu, A. et Lin, A. (2019). Translanguaging and trans-semiotising in a CLIL biology class in Hong Kong: whole-body sensemaking in the flow of knowledge co-making. Classroom Discourse, 10(3-4), 252-273.

https://doi.org/10.1080/19463014.2019.1629322 